\title{
Health Technology Assessments - Evidence Based Approach for A Sustainable Future of Healthcare
}

\author{
Manish Barman ${ }^{1 *}$, Narendra Kumar ${ }^{2}$ and Barman Palak ${ }^{3}$ \\ ${ }^{1}$ Department of Medicine, Hamad Medical Corporation, Assistant Professor of Clinical Medicine, Qatar
}

${ }^{2}$ Department of Cardiology, Manchester University NHS Foundation Trust, UK

${ }^{3}$ Vancouver School of Economics, Canada

*Corresponding author: Manish Barman, Department of Medicine, Hazm Mebaireek Hospital, Hamad Medical Corporation, Doha, Qatar

\section{ARTICLE INFO}

Received: 緊 July 31, 2019

Published: 幽 August 07, 2019

Citation: Manish Barman, Narendra Kumar, Barman Palak. Health Technology Assessments - Evidence Based Approach for A Sustainable Future of Healthcare. Biomed J Sci \& Tech Res 20(3)-2019. BJSTR. MS.ID.003440.

Keywords: Health Technology Assessment; Evidence Based Medicine

Abbreviations: HTA: Health Technology Assessments, CUA: Cost-Utility Analyses, EBM: Evidence Based Medicine, CEA: Cost-Effectiveness Analysis, CMA: Cost Minimization Analysis

\section{ABSTRACT}

Health Technology Assessments (HTA) are being increasingly used as an approach to guide and advise both pioneers and strategy creators for arrangement and delivery of medical services. This has been an after-effect of affirmation that resources are restricted, and consequently technologies need appraisals on standards past scientific favorability alone. HTA bodies require a precise audit of the huge clinical data for the technology and its comparators including systematic review of both asset utility and HRQOL information along with an extensive costeffectiveness data and critical appraisal of evidence on quality, current therapeutic standards and evidence-based medicine (EBM) guidance. The HTA practices vary considerably across technologies due to policy and decision-making criterions in varied political, economic and institutional context. It both represents and reflects approach and basic leadership in a specific geo-political scenario adopting methods and yields that fit the appropriate setting. HTA involve systematic and meticulous vetting of evidence in order to advise the policy makers or implement a change in any healthcare provision. Though there doesn't exist a simple single way to satisfy the needs of all stakeholders a balanced moderation is advised, especially in decentralised healthcare systems which engage multiple decision makers seeking to optimize value-based services subject to regional constraints. By adopting and incorporating few recommendations, HTA bodies can both enhance their credibility and accelerate technological development while also gaining greater public trust and support from health systems.

\section{Background}

Health Technology Assessments (HTA) are being increasingly used in numerous countries as an approach to guide and advise pioneers and strategy creators for arrangement of medical services [1-5]. This has been an after-effect of affirmation that resources are restricted, and consequently technologies need appraisals on standards past scientific favorability alone [6]. HTA organizations attempt to "streamline accessible assets" with "contextual, societal, and moral issues" [7]. While the bodies are widely similar to an extent, the distinction lies the meticulousness of the evaluation procedure and furthermore in the weightage and elucidation of the proof. HTA bodies consider broad impacts of any technology developed in light of unmet needs and innovation and tries to evaluate their potential and costs in both therapeutic and fiscal terms. It recognizes an intervention's optimal utilize, its appropriate position in the scope of care, and the patients who will benefit. The key components considered by any HTA during their decision making are as follows-

a. Effects on health indices: (QOL, death rates etc.,)

b. Adverse effects: however, this is viewed in context of available substitutes and innovation.

c. Disease burden: in the population with health, economic and social consequences 
d. Cost implications: on health provision and public-sector resources with QALY's gain.

e. Moral and communal connotations: accessibility, impartiality and equity.

f. Pertinence to population health needs: Effect on clinical practice and government policy prioritization.

g. Extent of novelty: addressing therapeutic alternatives

h. Unmet Needs: in a particular therapeutic area.

\section{Procedure / Approach}

HTA bodies require a precise audit of the huge clinical data for the technology and its comparators, including a systematic search strategy and progression of a "Quality of Reporting of Meta-Analysis" (QUOROM) flowchart. They also require systematic review of both asset utility and HRQOL information along with an extensive costeffectiveness data [8], a QUOROM explanation and critical appraisal of all significant RCT \& non-RCT evidence. However, risk sharing agreements and stakeholder's involvement can significantly vary across different bodies. HTA assessments are based on quality, current therapeutic standards and evidence based medicine (EBM) guidance (ISPOR 2014). This might include technological appraisals involving academia, health analysts, and healthcare providers [9]. The HTA practices vary considerably across technologies due to policy and decision-making criterions in varied political, economic and institutional context. It both represents and reflects approach and basic leadership in a specific political scenario utilizing methods and yields that fit the appropriate setting. All bodies have an unequivocal objective of applying a blend of both logical knowledge and communal values to decide on efficacy, safety of technologies in an impartial and straightforward way.

HTA bodies have clearly defined priority settings with implementation of rigorous, analytical methods for assessing costs and benefits in an evidence-based fashion in context to respective societal perspective. They all explicitly characterize uncertainty surrounding their estimates and acknowledge limitations while determining robustness of their findings and conclusions. All HTA bodies actively try to engage key stakeholders and answer issues of adaptability and interchangeability. However, the methodologies used to assess technologies, the austerity of the mechanisms, evidence analysis and consideration or weightage of other factors such as innovation, unmet needs and patient's choice clearly set out the differences amongst different HTA bodies.

\section{Discussion}

HTA are carried out with the aim to provide policy makers, key opinion leaders and stakeholders with evidence-based information on the relative cost and therapeutic benefits of the technology under consideration based on a meticulous assessment process. This helps decision makers to focus policies on value by maximizing health provisions for a given budget and gives patients and providers the information they need to select the best therapeutic options. However, the HTA process itself involves many issues which affect decision making criteria across technologies [10].

1) First, HTA itself can be impacted by the bodies and stakeholders involved in the process. HTA bodies differ in their remit and responsibilities but mostly work under government mandate along with independent entities. The extent of stakeholder involvement plays an important role in the process. While it enhances relevance and transparency of any HTA, it is resource and time intensive.

2) Second, although HTA bodies have a clear scientific and policy objective the methods adopted should be just as robust and transparent based on evidence and guidelines. However, this is not always true and concerns arise on selection and prioritization of areas of assessments, evidence selection, and review and data quality. HTA bodies should be country/province specific and often low-income areas lack resources to carry formal and detailed assessments.

3) Third, HTA impacts centers on its timeliness and effective implementation. National decisions might have issues at local levels and decisions can face challenges in a regional context where it might not be relevant or be a strain on local budgetary resources.

4) Fourth, health targets, funding, regional strategies and the inter sectoral collaboration with public health organizations, evaluation mechanisms all play an important role in decision making in different national settings across technologies.

5) Fifth, other parameters also affect and differ across technologies, such as

a) Primary Comparative Effectiveness Measurements (E.g. Some bodies evaluate primary clinical effectiveness as endpoints to constitute clinical dominance, analogy, or non-inferiority whereas others routinely factor in alternate outcomes from studies into decision making.)

b) Fiscal Assessment - may be cost-utility analyses (CUA) and/or cost-effectiveness analysis (CEA) and/or cost minimization analysis (CMA).

c) Other variables such as 'lack of alternative treatment' 'Unmet needs' or end of life considerations are also considered differently across technologies.

HTA are not just provisions for reimbursement decision making or a simple academic endeavour but also to inform about the importance and relevance of the technology. Early decisions guide us how to best adopt or optimise technology use for clinical practice. It is a critical amalgamation of many stakeholders (policy makers, practitioners, technology developers) to come to an agreement based on evidence and realistic terms. It is not a passive but an active facilitator of dialogue between stakeholders for optimal technology use throughout its life cycle. 


\section{Conclusion}

HTA involve systematic and meticulous vetting of evidence in order to advise the policy makers or implement a change in any healthcare provision. There doesn't exist a simple single way to satisfy the needs of all stakeholders and a balance line has to be drawn somewhere, especially in decentralised healthcare systems which engage multiple decision makers seeking to optimize valuebased services subject to regional constraints. However, despite restraints the HTA principles, structures and processes have a huge potential to provide cost effective \& efficient public healthcare. HTA enhances the credibility of innovation and technologies to accelerate in evolution of value-based provisions in general. HTA needs to be independent with clear objectives but it can afford to be defter and more flexible to broker alignment amongst developers, providers and users since the aim is to develop common understanding about potential innovation in improving public health outcomes without increasing intricacies for the public to comprehend [11]. This necessitates increasing participation of payers, providers, users and public to argue priorities, risk acceptance and trade off which in turn requires an exceptional harmonious arrangement to deliver this at the highest level.

\section{Recommendations}

Health service delivery is facing disruptive times. With extensive focus, priority and pressures, come a range of exciting opportunities. There is a need of collaborative evaluation amongst all stakeholders, be it providers, developers, regulators, academia, payers or HTA organizations to offer newer technologies and effective therapies to the world especially in areas of unmet needs with a balanced value perception. This shall involve earlier alignment of all stakeholders in the decision-making process, which technology to develop and what evidence to produce. This alignment would ensure that the process is smooth, effective and efficient in introduction of worthwhile technologies and rejecting ineffective technologies at the onset.

\section{Drug Developers}

i. Phase 1 should involve planned interactions with stakeholders to position conjectures and risks and raise discussions about indications, unmet needs, place in delivery pathway, expected benefits, regulatory approach and evidence generation requirements amongst market size and budget impacts.

ii. Phase 2 \& 3 should capture evidence generation in terms of population size, comparators, endpoints, benefits, generalizability issues, delivery optimizations and budget Impacts for optimal resource use. It can also include uncertainties and risk sharing potential.

iii. After phase 3, regulatory approvals, uncertainties and risk sharing potential can be detailed in light of the evidence generated including gaps if any.

\section{Device \& Other Technology Developers}

Device developers face issues like rapid evolution, no restrictions for aligned phase testing, user skills and training requirements and other business decisions. However, there is a similar need to fulfil evidence production and evaluation. This can be streamlined by process development involving regulatory and payer needs, identifying unmet needs, designing relevant studies for all stakeholders and synthesizing outcomes. Since innovation is mostly disruptive, evidence for improved patient outcomes and budgetary impacts should be aligned quite early amongst all stakeholders.

Technology developers can in general offer to explain their rationale for technology creation in a clear scientific manner for the stakeholders to formulate questions which will help developers in future evidence generation and cost benefit determinations. HTA bodies can act as facilitators amongst different stakeholders to engage early in a collaborative approach to healthcare delivery.

\section{HTA Bodies}

HTA bodies amalgamate important information for policy makers, clinical practitioners, users, developers \& health systems and synthesize evidence for all stakeholders to make informed choices. It facilitates understanding and expectations of all stakeholders in realistic and scientific terms. It absorbs multifaceted issues and presents guidance which is basic and fathomable to people in general to address their needs. However, HTA needs to assume a more dynamic role to assist and encourage discourse among all stakeholders to streamline innovation, improvement and utilization. Values change over time and HTA bodies should adopt a flexible approach throughout the whole lifecycle of technologies and develop shared perceptions for necessary evidence generation to prove value, enabling technology development.

a) The objectives and ambit of the HTA should be both unambiguous and specific, should include all relevant technologies and should be conducted as an unbiased and transparent exercise in a timely manner.

b) HTA should incorporate a clear prioritization based on national strategies and use standard methods for evaluating cost benefits.

c) Wide range of evidence and outcomes should be considered and include a societal perspective.

d) Uncertainties, concerns, generalizability and transferability should all be explicitly mentioned.

e) Should try best to incorporate all possible stakeholders to minimize bias.

f) Guidance should be clear and precise and communicated transparently to all stakeholders.

g) Recommendations should be monitored frequently after guidance is issued. 


\section{Process Improvement}

1. Data requirements should be aligned for both regulatory and advisory HTA bodies in line with reimbursement bodies.

2. Study designs should jointly fulfill the requirements of both regulators and payers.

3. Deft but flexible approach

4. HTA framework should be harmonized across international agencies by setting up standardized guidelines and definitions to address appropriate evidential requirements.

5. Wherein estimation of cost effectiveness is not adequate, provisional or conditional coverage decisions should be in place.

6. HTA need to decide research prospects and who should pay for it, public funding or developers?

7. Establish therapeutic or device-based registries for long term assessments and comparative analysis.

8. Use standardized protocols to address residual confounding.

9. Optimal diffusion of technology aligned with pay parity and funding mechanisms.

10. Bias adjustments from RCT's and observational studies

By adopting and incorporating few recommendations, HTA bodies can both enhance their credibility and accelerate technological development while also gaining greater public trust and support from health systems.

\section{References}

1. Drummond M, Sculpher M, O Brien B, Stoddart G, Torrance G, et al. (2007) Methods for the economic evaluation of health care programmes 3. New York: Oxford University Press.

\section{ISSN: 2574-1241}

\section{DOI: 10.26717/BJSTR.2019.20.003440}

Manish Barman. Biomed J Sci \& Tech Res

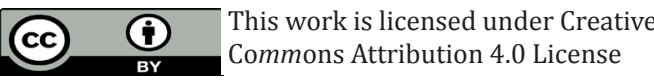

Submission Link: https://biomedres.us/submit-manuscript.php
2. Nielsen CP, Busse R (2008) Introduction In: Health Technology Assessment and Health Policy Making in Europe: Current status, challenges and potential. Garrido MV Kristensen FB Nielsen CP \& Busse R eds. World Health Organization (WHO).

3. Bergmann L, Enzmann H, Broich K, Hebborn A, Marsoni S, et al. (2014) Actual developments in European regulatory and health technology assessment of new cancer drugs: what does this mean for oncology in Europe? Annals of Oncology 25(2): 303-306.

4. Cairns J (2006) Providing guidance to the NHS: The Scottish Medicines Consortium and the National Institute for Clinical Excellence compared. Health Policy 76(2): 134-143.

5. Kanavos P, Nicod, Elena, Van den Aardweg S, Pomedli S, et al. (2010) Impact of health technology assessments: an international comparison. Euro Observer 12(4): 1-7.

6. Nicod Elena (2016) Why do health technology assessment coverage recommendations for the same drugs differ across settings? Applying a mixed methods framework to systematically compare orphan drug decisions in four European countries. The European Journal of Health. Economics 18(6): 715-730.

7. Drummond, Schwartz JS, Jönsson B, Luce BR, Neumann PJ, et al. (2008) Key principles for the improved conduct of health technology assessments for resource allocation decisions. International Journal of Technology Assessment in Health Care 24(3): 244-258.

8. Drummond M, Barbieri M, Cook J, Glick HA, Lis J, et al. (2009) Transferability of economic evaluations across jurisdictions: ISPOR Good Research Practices Task Force report. Value Health 12(4): 409418.

9. (2017) Healthcare Improvement Scotland. Scottish Medicines Consortium (SMC). What we do. Online.

10. Karen Facey, Chris Henshall, Laura Sampietro Colom, Sarah Thomas (2015) Improving the effectiveness and efficiency of evidence production for health technology assessment. Journal of Technology Assessment in Health Care 31(4): 201-206.

11. https://www.ispor.org/

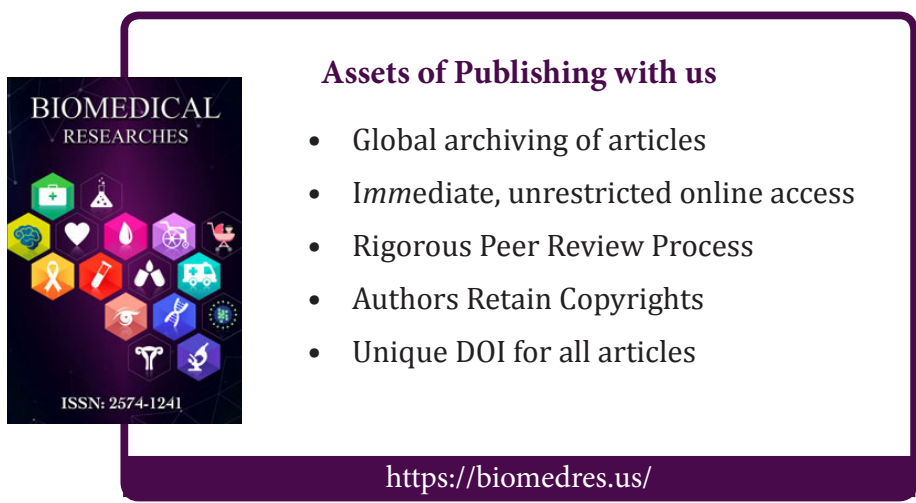

